\title{
Harpejamento em eqüinos no Rio Grande do Sul ${ }^{1}$
}

\author{
Aline Rodrigues ${ }^{2}$, Flavio D. De La Corte ${ }^{3}$, Dominguita L. Graça ${ }^{4}$, Daniel R. \\ Rissi $^{2}$, Ana L. Schild ${ }^{5}$, Glaucia D. Kommers ${ }^{4}$ e Claudio S.L. Barros ${ }^{4^{*}}$
}

\begin{abstract}
Rodrigues A., De La Corte F.D., Graça D.L., Rissi D.R., Schild A.L., Kommers G.D. \& Barros C.S.L. 2008. [Stringhalt in horses from the state of Rio Grande do Sul, Brazil.] Harpejamento em eqüinos no Rio Grande do Sul. Pesquisa Veterinária Brasileira 28(1):23-28. Departamento de Patologia, Universidade Federal de Santa Maria, 97105-900 Santa Maria, RS, Brazil. E-mail: claudioslbarros@uol.com.br

The epidemiology, clinical, gross and histological findings of cases of stringhalt occurring in horses from eight farms in six counties in the State of Rio Grande do Sul, Brazil from 2000-2005 are reported. At least 10 horses were affected. Ages of affected horses were 113 years (average 6.2 years) and 1-2 horses were affected in each farm. Factors that might have influenced the appearance of the disease included dearth of forage due to insufficient rainfall. The presence of the plant Hypochaeris radicata, often implicated as a cause of stringhalt in horses, was observed in the pasture of three out of five evaluated farms and in six of these farms the pasture was poor due to scarse precipitation. Estimated morbidity was $17.3 \%$ and lethality was close to zero although two horses were euthanatized for necropsy. Characteristic clinical signs included excessive flexion of the stifle and hock joints, impaired ambulation and bunny hop-type of gait. Clinical disease was graded by number scores from $1-5$, higher numbers indicating increasing severity. Three horses were graded as 1 , one horse as 2, three horses as 3, one horse as 4 and two horses as 5. Treatment with phenytoin in two horses and with phenytoin and tenectomy in another one did not result in amelioration of the clinical signs. Four out of ten clinical examined horses with stringhalt recovered with no treatment within 2-4 months of clinical disease and four affected horses did not recover even after 9-17 months of clinical disease, when they were lastly examined. Necropsy findings included atrophy of skeletal muscle of the large muscular groups which was confirmed histologically. Histological evaluation of peripheral nerves of one of the euthanatized horses revealed reduction or absence of myelinated fibers. Ultrastructural findings included signs of demyelination, regeneration and remyelination of peripheral nerves.
\end{abstract}

INDEX TERMS: Neuropathy, toxic neuropathy, diseases of horses.

RESUMO.- São relatados a epidemiologia, os sinais clínicos e aspectos macro e microscópicos de casos de harpejamento ocorridos de 2000 a 2005 em eqüinos de oito propriedades rurais de seis municípios do Rio Grande do Sul.

\footnotetext{
${ }^{1}$ Recebido em 15 de agosto de 2007.

Aceito para publicação em 28 de setembro de 2007.

2 Programa de Pós-Graduação em Medicina Veterinária, área de concentração em Patologia Veterinária, Centro de Ciências Rurais (CCR), Universidade Federal de Santa Maria (UFSM).

${ }^{3}$ Departamento de Clínica de Grandes Animais, CCR, UFSM, Santa Maria, RS 97105-900.

${ }^{4}$ Departamento de Patologia, UFSM, Santa Maria, RS 97105-900, Brasil. *Autor para correspondência: claudioslbarros@uol.com.br

${ }^{5}$ Laboratório Regional de Diagnóstico, Faculdade de Veterinária, Universidade Federal de Pelotas, 96010-900, Pelotas, RS.
}

Pelo menos 10 eqüinos foram afetados, com idades variando entre 1 e 13 anos (média de 6,2 anos) e 1-2 eqüinos foram afetados por propriedade. Dentre os fatores que podem ter influenciado o aparecimento da doença está incluída a escassez de forragem devido à seca. A presença da planta Hypochaeris radicata, freqüentemente implicada como causa de harpejamento em eqüinos, foi observada na pastagem de três entre cinco propriedades onde a ocorrência dessa planta foi investigada; em seis dessas propriedades a forragem era pouca devido à falta de chuva. A morbidade foi estimada em $17,3 \%$ e a letalidade foi perto de zero, embora dois eqüinos tenham sido submetidos à eutanásia para serem necropsiados. Os sinais clínicos característicos incluiam hiperflexão dos membros pélvicos, dificuldade de caminhar e andar com saltos tipo pulos de 
coelho. Foi feita uma graduação da intensidade dos sinais clínicos em um escore de números de 1 a 5 , os números mais altos indicando um grau de intensidade maior. Três eqüinos foram graduados como 1 , um eqüino como 2 , três eqüinos como 3 , um eqüino como 4 e dois eqüinos como 5. O tratamento com fenitoína em dois eqüinos e com fenitoína associada a tenectomia em um outro não resultou em melhora do quadro clínico. Quatro dos 10 cavalos com harpejamento examinados clinicamente se recuperaram sem tratamento após uma doença clínica com evolução de 2-4 meses e quatro cavalos não se recuperaram mesmo após 9-17 meses de doença clínica, quando foram examinados pela última vez. Os achados de necropsia incluíam atrofia dos músculos esqueléticos das grandes massas musculares, confirmada histologicamente. A avaliação histológica dos nervos periféricos de um eqüino afetado submetido à eutanásia revelou redução ou ausência de fibras mielinizadas. Os achados ultra-estruturais incluíam sinais de desmielinização, regeneração e remielinização de nervos periféricos.

TERMOS DE INDEXAÇÃO: Neuropatia, neuropatia tóxica, doenças de eqüinos.

\section{INTRODUÇÃO}

O harpejamento é uma síndrome de eqüinos caracterizada por andar anormal com flexão exagerada e involuntária de um ou ambos os membros pélvicos durante a movimentação (Pemberton \& Caple 1980). Duas formas são relatadas, o harpejamento convencional e o harpejamento australiano, que diferem entre si principalmente nos aspectos epidemiológicos. O harpejamento convencional tem ocorrência esporádica e etiologia desconhecida; nessa forma, os eqüinos afetados não se recuperam sem intervenção cirúrgica (Cahill \& Goulden 1992, Stashak 1994). O harpejamento australiano ocorre em surtos que atingem vários eqüinos de uma mesma propriedade ou região; embora ocorra principalmente na Austrália (Pemberton 1979, Pemberton \& Caple 1980, Robertson-Smith et al. 1985, Huntington et al. 1989, Slocombe et al. 1992), surtos dessa forma já foram descritos na Nova Zelândia (Cahill et al. 1985), no Chile (Araya et al. 1998) e nos Estados Unidos (Galey et al. 1991, Gay et al. 1993, Gardner et al. 2005). Os surtos geralmente iniciam no fim do verão e começo do outono, nos anos com longos períodos de seca e em eqüinos que tenham sido colocados em pastagens de má qualidade. A etiologia nesses casos ainda é obscura, porém, em muitos surtos foi observada uma relação com a presença abundante de uma planta (Hypochaeris radicata) na pastagem (Pemberton 1979, Cahill et al. 1985, Huntington et al. 1989, Gardner et al. 2005). Em geral, os casos da forma australiana apresentam recuperação espontânea após vários meses (Pemberton \& Caple 1980, Galey et al. 1991). No presente trabalho são descritos surtos de harpejamento em eqüinos no Rio Grande do Sul que ocorreram no período entre 2000 e 2005. Os dados epidemiológicos, clínicos e aspectos patológicos são apresentados.

\section{MATERIAL E MÉTODOS}

Os dados dos eqüinos com harpejamento foram obtidos por meio de entrevistas realizadas com proprietários e veterinários de cada propriedade, por visitas às propriedades e avaliação clínica dos eqüinos afetados. A intensidade do harpejamento foi graduada de 1-5 (sendo 1 o grau mais leve e 5 o mais acentuado) de acordo com parâmetros descritos para o harpejamento australiano (Huntington et al. 1989). Dois eqüinos que apresentavam um grau avançado da doença foram submetidos à eutanásia e necropsiados. Na necropsia desses eqüinos foram colhidos fragmentos de tecido de vários órgãos, que foram fixados em formol tamponado a $10 \%$ e processados rotineiramente para histologia. Fragmentos dos nervos periféricos foram fixados em glutaraldeído e processados para microscopia eletrônica.

\section{RESULTADOS}

Os casos de harpejamento ocorreram em 10 eqüinos de oito propriedades rurais de seis municípios do Rio Grande do Sul durante o inverno e a primavera de 2000-2005 (Quadro 1). O número de eqüinos afetados por propriedade variou de 1-2 e a idade dos animais era de 1-13 anos (média de 6,2 anos). Em seis propriedades foi possível colher informações tanto sobre o número de eqüinos

Quadro 1. Dados dos 10 eqüinos estudados nos surtos de harpejamento no Rio Grande do Sul

\begin{tabular}{|c|c|c|c|c|c|c|c|c|c|c|c|}
\hline Eqüino & Raça & Sexo & $\begin{array}{l}\text { Idade } \\
\text { (anos) }\end{array}$ & $\begin{array}{l}\text { Número de } \\
\text { eqüinos na } \\
\text { propriedade }\end{array}$ & $\begin{array}{l}\text { Número de } \\
\text { eqüinos } \\
\text { doentes }\end{array}$ & $\begin{array}{c}\text { Grau de } \\
\text { harpejamento }\end{array}$ & $\begin{array}{l}\text { Início dos } \\
\text { sinais } \\
\text { clínicos }\end{array}$ & $\begin{array}{c}\text { Duração } \\
\text { dos sinais } \\
\text { clínicos }\end{array}$ & Tratamento & $\begin{array}{c}\text { H. radicata } \\
\text { na pastagem }\end{array}$ & Município \\
\hline 1 & Misto & $\mathrm{Fb}^{\mathrm{b}}$ & 5 & $\mathrm{NIC}$ & $\mathrm{NI}$ & 3 & Out/00 & 3 meses & $N R^{d}$ & $\mathrm{NI}$ & Santo Ângelo \\
\hline 3 & PSC & $\mathrm{M}^{\mathrm{e}}$ & 1 & 2 & 1 & 5 & Ago/05 & 9 meses & $\begin{array}{l}\text { Fenitoína+ } \\
\text { tenectomia }\end{array}$ & Alta & Santa Maria \\
\hline 4 & Crioulo & $\mathrm{F}$ & 2 & 10 & 1 & 1 & Out/05 & 4 meses & NR & $\mathrm{NI}$ & Tupanciretã \\
\hline $7^{*}$ & Misto & $\mathrm{F}$ & 13 & 4 & 2 & 2 & Dez/05 & 2 meses & NR & Pouca & Bossoroca \\
\hline 8 & Crioulo & $\mathrm{F}$ & 7 & 3 & 1 & 4 & Dez/04 & 17 meses & NR & $\mathrm{NE}^{f}$ & Bossoroca \\
\hline $9^{* *}$ & Crioulo & $\mathrm{F}$ & 9 & 25 & 2 & 1 & Set/05 & 3 meses & NR & $\mathrm{NE}$ & São Borja \\
\hline $10^{* *}$ & Crioulo & $\mathrm{F}$ & 7 & 25 & 2 & 1 & Set/05 & 4 meses & NR & NE & São Borja \\
\hline
\end{tabular}

a Hypochaeris radicata; ${ }^{b}$ fêmea; ${ }^{c}$ não investigado; ${ }^{d}$ não realizado; ${ }^{\mathrm{e}}$ macho; ${ }^{\mathrm{f}}$ não encontrado; ${ }^{*} \mathrm{e}^{* *}$ pertencentes a uma mesma propriedade. 
afetados $(n=8)$ como o total de população eqüina sob risco nessas seis propriedades $(n=46)$, o que indica um coeficiente geral de morbidade de $17,3 \%$. Todos os casos ocorreram após períodos de seca. Em seis propriedades onde esse parâmetro foi avaliado a pastagem era de má qualidade. A planta $H$. radicata, apontada como causa do harpejamento (Huntington et al. 1989, Galey et al. 1991, Gardner et al. 2005), foi observada em grande quantidade em duas das cinco propriedades onde foi investigada, em pouca quantidade em uma propriedade e não foi encontrada nas outras duas. Em três propriedades, $50 \%$ dos eqüinos que tiveram acesso a $\mathrm{H}$. radicata adoeceram. Dos 10 eqüinos afetados, sete eram da raça Crioula, um da raça Puro Sangue de Corrida e dois eram mistos; oito eqüinos eram fêmeas e dois eram machos. Os graus de intensidade clínica do harpejamento desses 10 eqüinos está no Quadro 1. Três eqüinos apresentaram Grau 1 de intensidade da manifestação clínica de harpejamento; esses eqüinos mostravam hiperflexão dos membros pélvicos apenas quando andavam em círculos, para trás ou quando eram estressados. Um eqüino classificado como Grau 2 apresentava movimentos esporádicos de harpejamento quando movimentado ao passo ou ao trote. O Grau 3, observado em três eqüinos, caracterizavase por hiperflexão moderada dos membros pélvicos, que
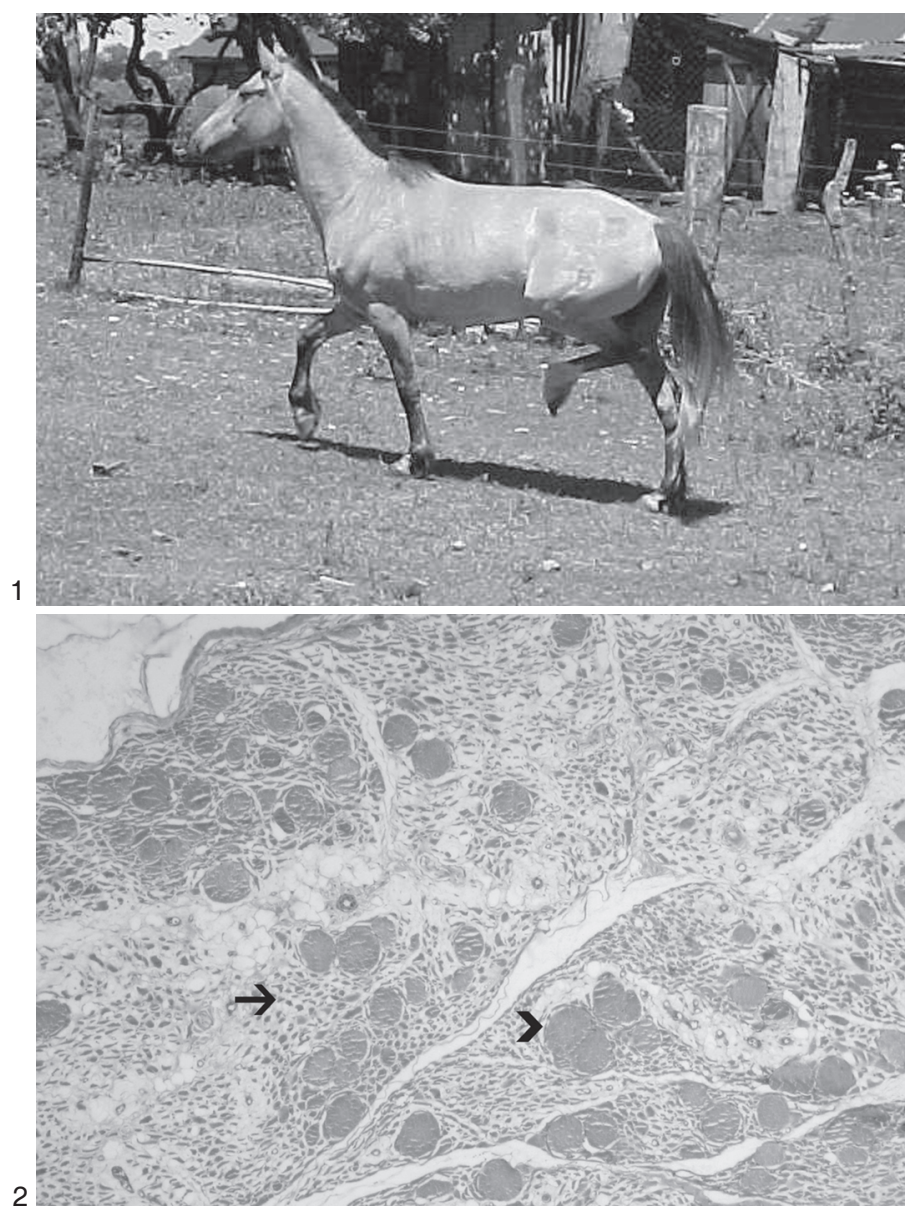

ocorria ao passo, ao trote e, especialmente, ao salto. $\mathrm{O}$ Grau 4 foi observado em um eqüino que apresentava hiperflexão acentuada dos membros pélvicos (que tocavam o abdômen) com o eqüino em estação, andando ou correndo. O Grau 5 de harpejamento foi observado em dois eqüinos e caracterizava-se por hiperflexão dos membros pélvicos (Fig.1) por vários segundos, difícil movimentação e saltos semelhante aos de coelho com os dois membros pélvicos. Além desses sinais, os eqüinos afetados não conseguiam andar para trás ou em círculos. Três eqüinos foram tratados com fenitoína na dose de $15 \mathrm{mg} /$ $\mathrm{kg}$ durante 20-60 dias. Em um desses eqüinos foi realizada miotenectomia do nervo extensor digital lateral. Em nenhum dos casos observou-se melhora do quadro clínico após o tratamento.

Dos 10 eqüinos avaliados, quatro (Eqüinos 4, 7, 9 e 11) se recuperaram totalmente após evolução clínica de 2-4 meses, mesmo sem tratamento. Desses quatro, três tinham Grau 1 de harpejamento e um tinha Grau 2. Quatro eqüinos (Eqüinos 3, 5, 6 e 8) não se recuperaram e apresentavam sinais da doença na última vez que foram examinados, 9-17 meses após o início dos sinais clínicos. Os Eqüinos 1 e 2 foram submetidos à eutanásia e necropsiados com três meses de evolução do quadro de harpejamento. Ambos tinham atrofia acentuada dos músculos dos membros pélvicos e da garupa. Um desses eqüinos apresentava fraturas nas costelas. Histologicamente observou-se que os músculos semitendináceo (Fig.2), quadríceps femoral, bíceps femoral, glúteo superficial, glúteo médio e sacrocaudal dorsal médio do Eqüino

Fig.1. Eqüino 2, com sinais clínicos de harpejamento, mostrando hiperflexão do membro pélvico esquerdo.

Fig.2. Músculo semitendináceo do Eqüino 1, com fibras atróficas (setas) em meio a fibras normais (cabeças de setas). HE, obj.20x.

Fig.3. Nervo laríngeo recorrente do Eqüino 2, com harpejamento. Observam-se raras fibras largas mielinizadas nos fascículos do nervo. Azul de toluidina, obj.100x.

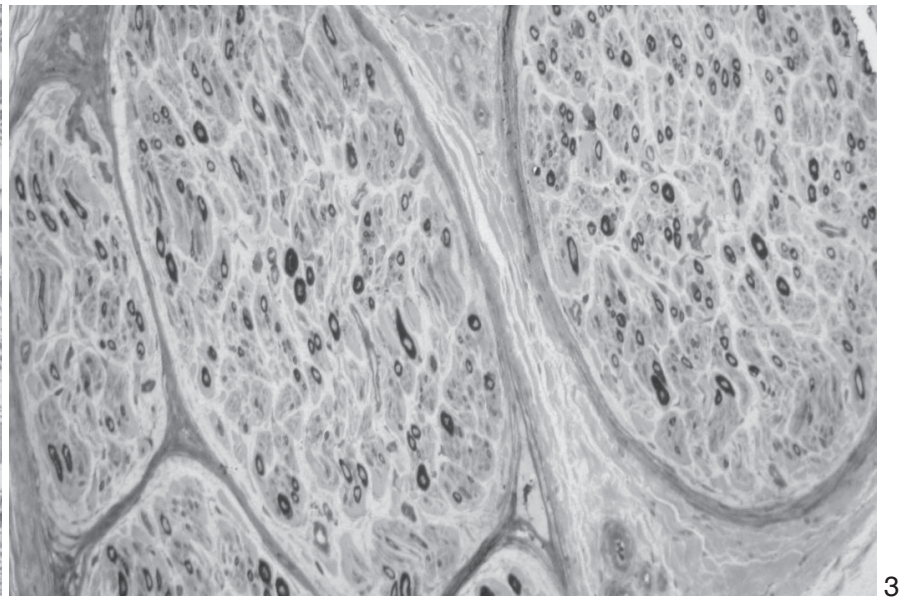




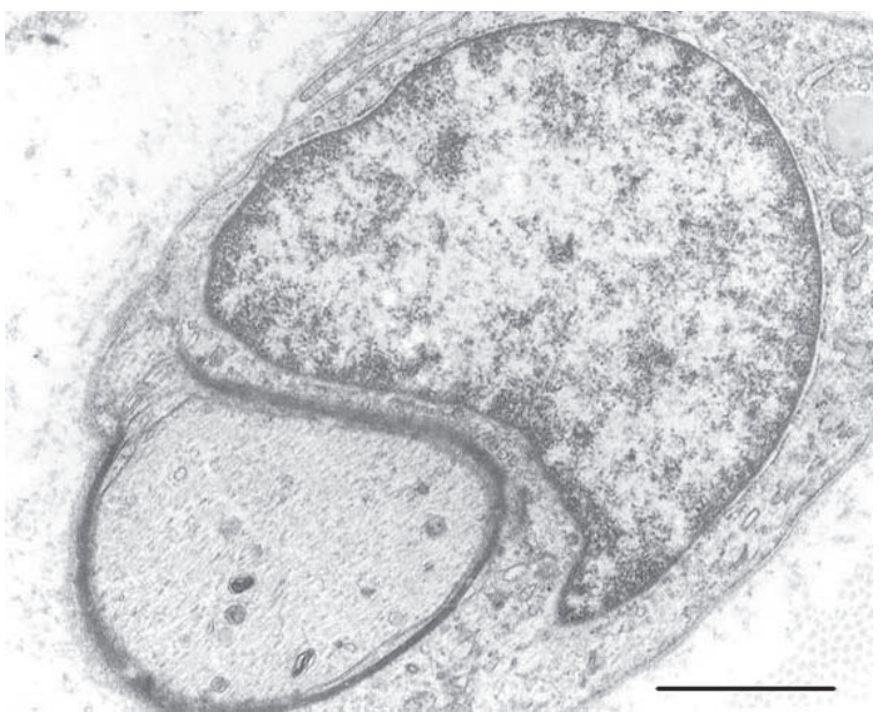

Fig.4. Nervo periférico do Eqüino 2 com harpejamento. Axônio em processo de remielinização envolvido pela célula de Schwann (CS). A imagem mostra o centro de um internodo, caracterizado pela presença do núcleo da CS. A membrana basal é descontínua e há abundantes fibras colágenas no endoneuro. Microscopia eletrônica, barra $=1 \mu \mathrm{m}$.

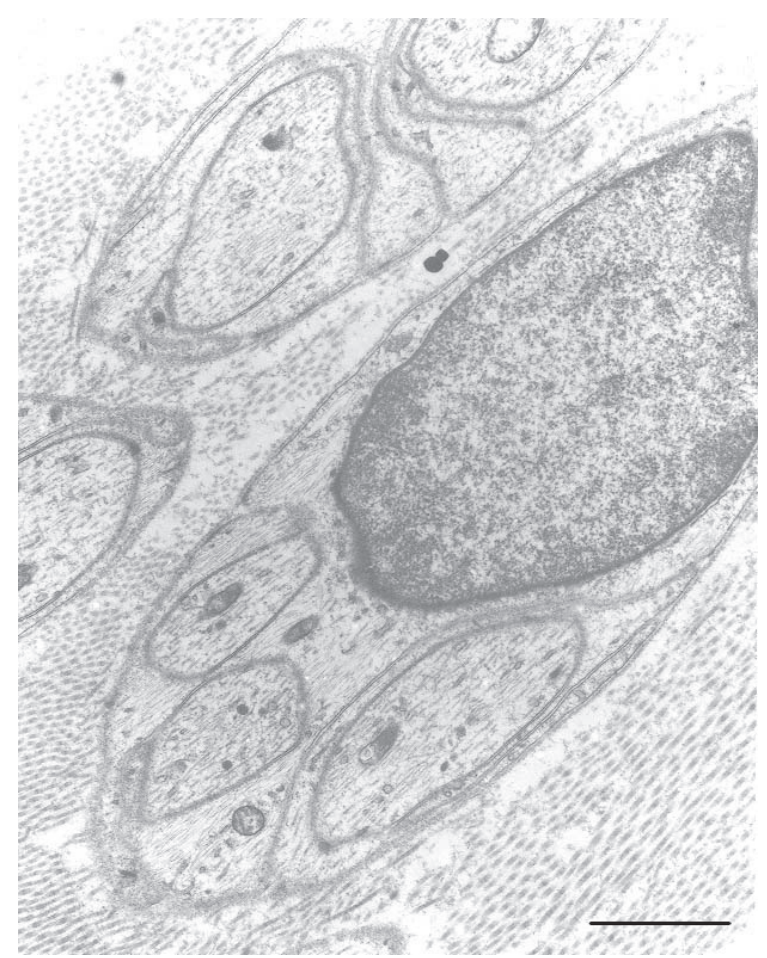

Fig.5. Nervo periférico do Eqüino 2 com harpejamento. Vários neuritos são envolvidos por uma célula de Schwann. $\mathrm{Na}$ imagem à direita é observado o núcleo de uma delas. A membrana basal é conspícua. Microscopia eletrônica, bar$\mathrm{ra}=1 \mu \mathrm{m}$.

2 apresentavam degeneração e necrose muscular leve a moderada; no músculo quadríceps femoral a atrofia era acentuada.

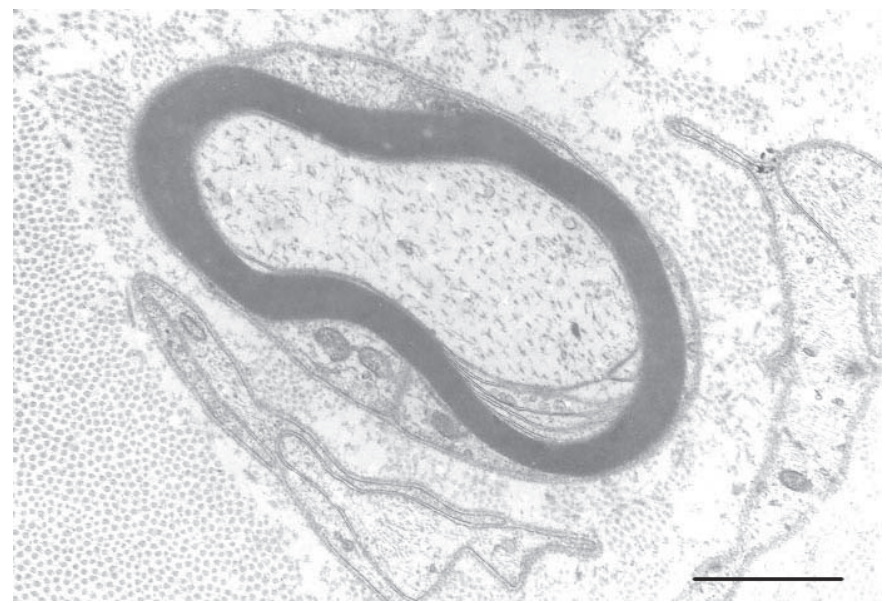

Fig.6. Nervo periférico do Eqüino 2 com harpejamento. Axônio remielinizado. Há processos de uma célula de Schwann dispostos concentricamente ao redor e entremeados com abundantes fibras colágenas no endoneuro. Microscopia eletrônica, barra $=1 \mu \mathrm{m}$.

Cortes semifinos corados com azul de toluidina dos nervos laríngeo recorrente e tibial distal do Eqüino 1 demonstraram uma redução no número de fibras mielinizadas (Fig.3) e diminuição do número de fibras. Ultra-estruturalmente observaram-se regeneração e remielinização nos nervos periféricos (Fig.4); alguns axônios, contudo, ainda não possuiam bainha. Numerosos processos das células de Schwann envolviam axônios e neuritos de pequeno diâmetro (Fig.5). Restos de mielina podiam ser encontrados no citoplasma das células de Schwann. No endoneuro havia colágeno em abundância (Fig.6). Não foram observadas alterações nos demais nervos coletados.

\section{DISCUSSÃO}

Todos os casos descritos aqui ocorreram em anos onde se registraram longos períodos de seca e em eqüinos mantidos em pastagens de má qualidade, aspectos epidemiológicos semelhantes aos descritos para o harpejamento australiano (Pemberton \& Caple 1980, Huntington et al. 1989, Cahill \& Goulden 1992). Na Austrália, Nova Zelândia e Estados Unidos, $H$. radicata tem sido associada com casos de harpejamento (Huntington et al. 1989, Galey et al. 1991, Gardner et al. 2005). Essa planta cresce bem em solos pobres e inférteis e pode suprimir a pastagem. No verão, suas flores têm um cabo longo e os eqüinos comem as flores, os caules e as foIhas (Huntington et al. 1989, Burrows \& Tyrl 2001). Nos surtos de harpejamento descritos neste trabalho, $\mathrm{H}$. radicata foi observada na maioria das propriedades (Cahill et al. 1985, Huntington et al. 1989, Galey et al. 1991, Gay et al. 1993), embora alguns surtos ocorreram em propriedades onde essa planta não foi observada ou a quantidade observada não era significativa (Robertson-Smith et al. 1985, Araya et al. 1998). As explicações para isso podem ser: (1) a planta pode não estar envolvida na etiologia; (2) a planta e outras causas podem estar envolvidas; e 
(3) $H$. radicata pode ser a única causa e os eqüinos de propriedades onde não havia a planta tiveram acesso a $H$. radicata de outra maneira, i.e., pela ingestão de feno de alfafa contaminado pela planta. Embora essa possibilidade não tenha sido por nós investigada, é mencionado que no noroeste do Rio Grande do Sul $H$. radicata freqüentemente contamina a alfafa destinada a feno durante a colheita (Jardim Filho 2006). Por outro lado, nem todos os eqüinos mantidos em pastagens infestadas por H. radicata adoecem (Pemberton \& Caple 1980, Huntington et al. 1989) e tentativas de intoxicação experimental pela administração da planta a eqüinos têm falhado (Cahill \& Goulden 1992). Tem sido sugerido que um período de seca prolongada poderia ser o principal fator predisponente para a doença por permitir o desenvolvimento de uma condição carencial, ou ainda, que a produção de micotoxinas no solo ou nas próprias plantas seja a causa; no entanto, fungos que possam produzir essas micotoxinas não têm sido identificados (Pemberton \& Caple 1980, Cahill et al. 1986, Slocombe et al. 1992). Em resumo, embora os casos de harpejamento sejam observados há muitos anos, ainda não se determinou a causa (ou causas) da doença. Nos surtos aqui relatados foram observados alguns fatores envolvidos na epidemiologia da doença (Robertson-Smith et al. 1985, Huntington et al. 1989, Gay et al. 1993, Araya et al. 1998), como a predominância de estações mais secas nos anos em que os surtos ocorrem e pastagens de má qualidade e escassas. Em geral, os casos de harpejamento são comumente descritos no fim do verão e outono, embora relatos descrevem a primavera como a época de maior ocorrência dos casos (Hungerford 1975).

O número de eqüinos afetados por propriedade variou consideravelmente e, em geral, apenas um ou dois eqüinos eram afetados por propriedade, semelhante ao que foi anteriormente observado nos casos de harpejamento em eqüinos na Austrália (Huntington et al. 1989).

A hiperflexão geralmente é bilateral nos membros pélvicos, sendo raramente unilateral (Pemberton 1979, Robertson-Smith et al. 1985, Huntington et al. 1989). Ocasionalmente os quatro membros podem estar afetados e os eqüinos que chegam a essa fase geralmente são sacrificados (Huntington et al. 1989). O grau de severidade pode variar entre os membros, principalmente na fase aguda e na fase de recuperação (Cahill et al. 1986, Robertson-Smith et al. 1985, Huntington et al. 1989). Em todos os eqüinos deste relato a hiperflexão ocorreu bilateralmente nos membros pélvicos. É descrito que eqüinos afetados por harpejamento podem também desenvolver hemiplegia laríngea, devida a lesões no nervo laríngeo recorrente (Pemberton \& Caple 1980, Huntington et al. 1989, Slocombe et al. 1992), mas esse sinal clínico não foi observado nos eqüinos deste estudo.

Um fato comumente observado nos casos de harpejamento australiano é a recuperação dos sinais clínicos mesmo sem terapia ou intervenção cirúrgica
(Pemberton 1979, Pemberton \& Caple 1980, Cahill et al. 1985), em eqüinos que são removidos para um campo de melhor qualidade (Cahill et al. 1985, Huntington et al. 1989, Gay et al. 1993, Araya et al. 1998, Gardner et al. 2005). Nesses casos, o período de recuperação pode variar de poucos dias até mesmo vários anos, mas, em geral, o quadro clínico melhora em 6-12 meses (Huntington et al. 1989, Cahill \& Goulden 1992). Nos eqüinos deste estudo, o período de recuperação variou de 2 meses até mais de um ano, sendo que quatro eqüinos não se recuperaram.

Nos casos em que se preconiza a realização de um tratamento, é indicado o uso de fenitoína na dose de 15 $\mathrm{mg} / \mathrm{kg}$, um anticonvulsivante de ação central (Huntington et al. 1991), ou a realização da miotenectomia do extensor digital lateral (Huntington et al. 1989, Mayhew 1989). Quando a miotenectomia do extensor digital lateral é realizada, espera-se que ocorra recuperação em $50 \%$ dos casos. Nos demais casos a cirurgia pode não funcionar, pois a flexão do jarrete também resulta da ação dos músculos extensor digital longo e tibial anterior, que estão sempre lesionados nos casos de harpejamento (Huntington et al. 1989). O uso da fenitoína traz resultados satisfatórios durante a administração do medicamento, porém quando o tratamento é interrompido os sinais clínicos podem retornar em um grau mais leve (Huntington et al. 1989). Além disso, o uso da fenitoína pode diminuir o tempo de recuperação dos sinais clínicos. Nos três eqüinos deste relato em que a fenitoína foi administrada não houve meIhora no quadro clínico, sendo que dois desses eqüinos permaneceram doentes por longos períodos e o outro eqüino teve que ser eutanasiado devido ao avançado grau de lesão. O período de recuperação dos sinais clínicos vai depender do grau de lesão dos nervos e da habilidade dos axônios em se regenerar. Quanto mais extensa a injúria ao nervo, mais longo será o período de recuperação. Devido às longas distâncias que os axônios em regeneração deverão percorrer, a recuperação será um processo lento e talvez até incompleto (Cahill et al. 1986).

A atrofia muscular observada nos eqüinos deste relato é secundária à neuropatia periférica e ocorre por desnervação muscular. Em geral, os músculos extensor digital lateral, tibial cranial, cricoaritenóide dorsal, grácilis e flexor digital profundo lateral são os mais afetados e apresentam atrofia acentuada das fibras musculares e fibrose difusa com variação no tamanho e na forma das fibras musculares. Nos músculos menos afetados podese observar variação no tamanho e na forma das fibras musculares (Slocombe et al. 1992).

As lesões de nervo periférico observadas no Eqüino 1 são características de uma axonopatia distal. Os nervos, quando observados em cortes semi-finos, evidenciaram lesões crônicas caracterizadas principalmente por regeneração das fibras nervosas. Embora o Eqüino 2 apresentasse clinicamente um grau acentuado de harpejamento, não foram observadas alterações microscópicas nas amostras de nervos examinadas. Uma possível explicação para isso é que as lesões observadas 
nos casos de harpejamento são mais acentuadas na porção distal dos nervos (Cahill et al. 1986, Slocombe et al. 1992), e nesse caso, apenas a porção proximal foi coletada. Os nervos mais comumente afetados são o peroneal superficial, peroneal profundo, tibial distal, digital plantar, volar e o nervo laríngeo recorrente (Slocombe et al. 1992). É possível que a doença sempre envolva destruição total do axônio periférico, porque a degeneração inicia-se nas partes mais distais dos nervos e a porção proximal, nesses casos, deve ser preservada para que ocorra a regeneração. Em tais circunstâncias, regeneração e recuperação total da função ocorrem mais rapidamente (Slocombe et al. 1992).

Ultra-estruturalmente, as principais alterações observadas nos nervos caracterizavam um processo crônico em fase de regeneração, i.é, remielinização em andamento, neuritos envolvidos por células de Schwann e proliferação concêntrica de células de Schwann ao redor de axônios remielinizados. Em uma fase inicial, pode-se observar degeneração walleriana das fibras de maior diâmetro, grande número de células de Schwann desnervadas e debris de mielina. Atrofia das fibras nervosas pode também ocorrer e caracteriza-se por fibras com bainhas de mielina desproporcionalmente espessadas em relação ao seu calibre axonal (Cahill et al. 1986).

Muitos estudos foram realizados para determinar a patogênese dos casos de harpejamento. Alguns autores sugerem que essa seja uma alteração primária dos nervos, enquanto outros apontam para a possibilidade de uma lesão encefálica estar associada (Pemberton \& Caple 1980), mas até a presente data não foi possível definir a patogênese dessa condição. Novos estudos são necessários para estabelecer a epidemiologia, etiologia e patogênese dos casos de harpejamento.

Um quadro de axonopatia distal é relatado em várias espécies animais após intoxicação tardia por organofosforados (OF) (Souza et al. 1996). Em cavalos, a lesão do nervo laríngeo recorrente com aparecimento de rouquidão pode ser idiopática ou associada com intoxicação por OF (Rose et al. 1981). Nesse quadro, alguns autores relatam a ocorrência de uma polineuropatia (Cahill \& Goulden 1986) cujo aspecto histológico e ultra-estrutural é o de degeneração walleriana, melhor observada pelo método das fibras desfiadas (Souza et al. 1996), concomitantemente com a regeneração de fibras, a presença de CS supranumerárias e abundância de colágeno no endoneuro, como observado nos cavalos deste relato. $\mathrm{Na}$ intoxicação experimental em ovinos, diferentemente do que é observado no harpejamento, as lesões periféricas na intoxicação tardia por OF são acompanhadas de lesões do SNC com agravamento progressivo do quadro clínico de flacidez muscular e mau prognóstico para os animais afetados (Souza et al. 1996). Alguns relatos de harpejamento descrevem diminuição dos sinais neurológicos até a cura espontânea, como aconteceu nos Eqüinos 4, 7, 9 e 11 deste estudo.

\section{REFERÊNCIAS}

Araya O., Krause A. \& Solis de Ovando M. 1998. Outbreaks of stringhalt in Southern Chile. Vet. Rec. 142:462-463.

Burrows G.E. \& Tyrl R.J. 2001. Asteraceae Dumort, p.147-254. In: Ibid. (ed.), Toxic Plants of North America. lowa State University Press, Ames. 1342p.

Cahill J.I. \& Goulden B.E. 1986. Equine laryngeal hemiplegia. Part I. A light microscopic study of peripheral nerves. N.Z. Vet. J. 34:161-169.

Cahill J.I. \& Goulden B.E. 1992. Stringhalt: current thoughts on aetiology and pathogenesis. Equine Vet. J. 24:161-162.

Cahill J.I., Goulden B.E. \& Pearce H.G. 1985. A review and some observations on stringhalt. N.Z. Vet. J. 33:101-104.

Cahill J.I., Goulden B.E. \& Jolly R.D. 1986. Stringhalt in horses: a distal axonopathy. Neuropathol. Appl. Neurobiol. 12:459-475.

Galey F.D., Hullinger P.J. \& McCaskill J. 1991. Outbreaks of stringhalt in Northern California. Vet. Hum. Toxicol. 33:176-177.

Gardner S.Y., Cook A.G., Jortner B.S., Troan B.V., Sharp N.J.H., Campbell N.B. \& Brownie C.F. 2005. Stringhalt associated with a pasture infested with Hypochaeris radicata. Eq. Vet. Ed. 7:154-158.

Gay C.C., Fransen S., Richards J. \& Holler S. 1993. Hypochaerisassociated stringhalt in North America. Eq. Vet. J. 25:456-457.

Hungerford T.G. 1975. Diseases of Livestock. 8th ed. McGraw-Hill, Sydney.

Huntington P.J., Jeffcott L.B., Friend S.C.E., Luff A.R., Finkelstein D.I. \& Flynn R.J. 1989. Australian stringhalt: epidemiological, clinical and neurological investigations. Eq. Vet. J. 21:266-273.

Huntington P.J., Seneque S., Slocombe R.E, Jeffcott L.B., McLean A. \& Luff A.R. 1991. Use of phenytoin to treat horses with Australian stringhalt. Aust. Vet. J. 68:221-224.

Jardim Filho J.O. 2006. Comunicação pessoal (Depto Clínica de Grandes Animais, Universidade Federal de Santa Maria, 97105-900 Santa Maria, RS).

Mayhew I.G. 1989. Opisthotonus, tetanus, myoclonus, tetany, tremor, and other localized muscle spasms and movement disorders, p.197225. In: Ibid. (ed.), Large Animal Neurology: A handbook for veterinary clinicians. Lea and Febiger, Philadelphia. 380p.

Pemberton O.H. 1979. Investigations of a recent outbreak of stringhalt in horses in East Gippsland. Vic. Vet. Proc. 37:16.

Pemberton O.H. \& Caple I. W. 1980. Australian stringhalt in horses. Vet. Ann. 20:167-171.

Robertson-Smith R.G., Jeffcott L.B., Friend S.C.E. \& Badcoe L.M. 1985. An unusual incidence of neurological disease affecting horses during a drought. Aust. Vet. J. 62:6-12.

Rose R.J., Hartley W.J. \& Baker W. 1981. Laryngeal paralysis in Arabian foals associated with oral Haloxon administration. Equine Vet. J. 13:171-176

Slocombe R.E, Huntington P.J., Friend S.C.E., Jeffcott L.B., Luff A.R. \& Finkelstein O.K. 1992. Pathological aspects of Australian stringhalt. Eq. Vet. J. 24:174-183.

Souza M.V., Graça D.L., Ferrão S.M.N. \& Contesini E.A. 1996. Regeneration of peripheral nerves fibers following Haloxon-induced degeneration. Braz. J. Vet. Res. Anim. Sci. 33:231-234.

Stashak T.S. 1994. Harpejamento, p.755-756. In: Ibid. (ed.), Claudicação em Eqüinos segundo Adams. 4ª ed. Roca, São Paulo. 943p. 